\title{
A THEOREM ON METRIZATION OF MOORE SPACES
}

\section{J. N. YOUNGLOVE}

R. H. Bing has shown [1, Theorem 8] that a normal, screenable Moore space is metrizable. This paper is devoted to showing that the same conclusion follows by replacing screenability in the hypothesis by the assumption that boundaries of domains are screenable and by adding the requirement of completeness on the space.

The statement that the space $\Sigma$ is a complete Moore space means that there is a sequence of collections of regions in $\Sigma$ satisfying Axiom 0 and all of Axiom 1 of [2].

The statement that the point set $M$ is screenable means that if $G$ is an open covering of the space $\Sigma$, then there is a sequence $\left\{H_{i}\right\}$ of collections of disjoint open sets such that $\sum H_{i}$ is a refinement of $G$ which covers $M$. The sequence $\left\{H_{i}\right\}$ is said to screen $M$ with respect to $G$.

Theorem. A normal, complete Moore space is screenable, thus metrizable, provided that the boundary of each domain is screenable.

Proof. Let $G$ denote an open covering of a normal, complete Moore space $\Sigma$. There is by [3] a metrizable subspace $\Sigma^{\prime}$ of $\Sigma$ such that $\Sigma^{\prime}$ is dense in $\Sigma$. Let $G^{\prime}$ denote a covering of $\Sigma^{\prime}$ such that if $g^{\prime}$ is in $G^{\prime}$, then there is an element $g$ of $G$ such that $g \cdot \Sigma^{\prime}$ is $g^{\prime}$. In the space $\Sigma^{\prime}, G^{\prime}$ is an open covering of $\Sigma^{\prime}$. By a theorem of Bing [1, Theorem 3] there is a sequence $\left\{H_{i}^{\prime}\right\}$ of discrete collections of open sets (open in $\Sigma^{\prime}$ ), such that the sum of the collections of the sequence is a refinement of $G^{\prime}$ which covers $\Sigma^{\prime}$. Let $h^{\prime}$ denote an element of some $H_{i}^{\prime}$. Let $g^{\prime}$ denote a set in $G^{\prime}$ such that $h^{\prime}$ is a subset of $g^{\prime}$. There is a set $g$ in $G$ such that $g \cdot \Sigma^{\prime}$ is $g^{\prime}$. There is a domain $D$ in $\Sigma$ such that $D \cdot \Sigma^{\prime}$ is $h^{\prime}$. Let $h$ denote the set $D \cdot g$. The set $h$ is a domain in $\Sigma$ which is a subset of $g$ and whose intersection with $\Sigma^{\prime}$ is $h^{\prime}$. Let $H_{i}$ denote a collection such that for each $h^{\prime}$ in $H_{i}^{\prime}$ there is exactly one $h$ in $H_{i}$ for which $h \cdot \Sigma^{\prime}$ is $h^{\prime}$. Suppose that some two elements $d_{1}$ and $d_{2}$ of $H_{i}$ intersect. The set $d_{1} \cdot d_{2}$ is an open set and must therefore intersect $\Sigma^{\prime}$ since $\Sigma^{\prime}$ is dense in $\Sigma$. But $d_{1} \cdot \Sigma^{\prime}$ is an element of $H_{i}^{\prime}$ and $d_{2} \cdot \Sigma^{\prime}$ is a different element of $H_{i}^{\prime}$. It follows that $H_{i}$ is a collection of disjoint sets.

Let $H$ denote the logical sum of all the collections in the sequence $H_{i}$. The set $H^{*}$ is a domain which contains $\Sigma^{\prime}$. Thus $H^{*}$ is dense in $\Sigma$.

Presented to the Society, November 25, 1960 under the title Two theorems on metrization of Moore spaces; received by the editors August 26, 1960. 
If $H^{*}$ is $\Sigma$, then the sequence $\left\{H_{i}\right\}$ screens $\Sigma$ with respect to $G$. If $H^{*}$ is not $\Sigma$, then there is a sequence $\left\{K_{i}\right\}$ of open sets which screens the boundary of $H^{*}$ with respect to $G$. The sequence $H_{1}, K_{1}, H_{2}$, $K_{2}, \cdots$ screens $\Sigma$ with respect to $G$. This completes the proof that $\Sigma$ is screenable. Theorem 8 of [1] assures the metrizability of $\Sigma$.

\section{REFERENCES}

1. R. H. Bing, Metrization of topological spaces, Canad. J. Math. vol. 3 (1951) pp. 175-186.

2. R. L. Moore, Foundations of point set theory, Amer. Math. Soc. Colloquium Publications, vol. 13, 1932.

3. J. N. Younglove, Concerning dense metric subspaces of certain nonmetric spaces, Fund. Math. vol. 48 (1959) pp. 15-25.

UNIVERSITY OF MisSOURI 